\title{
A New Semiempirical Approach to Study Ground and Excited States of Metal Complexes in Biological Systems ${ }^{\dagger}$
}

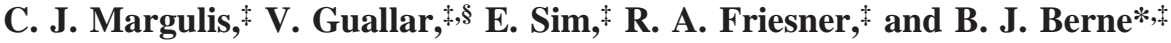 \\ Department of Chemistry, Columbia University, 3000 Broadway, New York City, New York 10027, and \\ Department of Chemistry, University of California at Berkeley, Berkeley, California
}

Received: March 13, 2002; In Final Form: June 4, 2002

\begin{abstract}
In this paper we develop a "diatomic in molecules semiempirical ligand field" (DIMSELF) method to calculate ground and excited many-body potential energy surfaces for an arbitrary transition metal ion in an arbitrary complex system. This method is not restricted to a high-symmetry environment and is meant to be inexpensive and suitable for nonadiabatic excited states dynamics on-the-fly. Within the approximations employed, the method includes full CI (configuration interaction) and SO (spin-orbit) interactions, essential to the description of nonradiative transitions such as those of myoglobin in the presence of carbon monoxide. We test our method against high level ab initio calculations for a simple model system of myoglobin's heme pocket. Finally, we discuss our results and compare with previous calculations in the literature.
\end{abstract}

\section{Introduction}

Transition metal complexes play a fundamental role in many technological and biological areas. They act as catalysts in the oil industry during the processes of cracking and reforming, they are also fundamental for oxygen transport in blood, and they are key to plant respiration. Some of these processes can be well understood without the need to include the small contribution of spin-orbit (SO) interaction which, for the most part, is of the order of the error one performs in ab initio calculations of these kind of systems. In some cases, however, nonradiative transitions occur between states with different spins. Of particular interest are heme proteins, a biologically important group of molecules that have a unique common active site: an iron-protoporphyrin-IX complex, where changes in spin state can be an integral part of the protein function. One of the most striking biological manifestations of this modulation is the binding of molecular oxygen and carbon monoxide $(\mathrm{CO})^{1-9}$ to the five-coordinated ferrous heme site of globins. Another wellstudied example is the situation of several spin changes in the enzymatic activation cycles of cytochromes P450. These transitions are solely due to $\mathrm{SO}$ coupling and are otherwise forbidden.

$\mathrm{Ab}$ initio calculations are performed within the framework of Hund's Case A and hence assume that S, the total spin of the system, is a good quantum number. Spin-orbit coupling matrix elements could then be added, and rediagonalization of the Hamiltonian matrix would provide us with the spin coupled eigenstates of the system. Obtaining several electronic excited states for a given spin multiplicity is complex, and for mediumsized systems only configuration interaction for single excitations (CIS) is affordable. However, a more complete description of excited states might be required for the study of dynamics. For large systems, where the environment can be introduced by mixing quantum chemistry with molecular mechanics, electronically excited states are rarely computationally accessible

\footnotetext{
† Part of the special issue "John C. Tully Festschrift".

* Corresponding author.

$\doteqdot$ Columbia University.

$\S$ University of California at Berkeley.
}

and only the fundamental ground state for each spin multiplicity might be computed. For nonsymmetric systems, even at the density functional theory (DFT) level of theory, several attempts with different initial guesses should be considered in order to achieve the true ground state. The computational effort is drastically increased when several nuclear configurations are required for a complete study of the dynamics, and calculations on-the-fly, including excited states and SO coupling, are simply out of the question. Therefore, a semiempirical approach that includes excited states and SO coupling is very desirable.

The method we develop in this paper is a generalization of Gerloch's ${ }^{10}$ cellular AOM (angular overlap model) version of ligand field theory, combined with a semiempirical version of Ellison's ${ }^{11-13}$ DIM (diatomics in molecules). Neither ligand field theory nor the semiempirical version of DIM are exact ab initio methods; not all the parameters can be obtained by analytical methods and they ought to be fitted either to experimental data or other calculations. On the other hand, once these parameters are known, fast computation of ground- and excited-state potentials can be performed without having to recalculate wave functions and their matrix elements.

We test this method against a simplified model of the heme pocket. This reduced system, shown in Figure 1, is composed of the $\mathrm{Fe}$ (II) ion, the heme ring, the $\mathrm{CO}$ molecule, and a histidine residue. Others have used the same system ${ }^{14,15}$ to quantify the size of potential energy barriers between ground-state quintet and ground-state singlet and make predictions about reaction rates. It is known that in the absence of $\mathrm{CO}$ the electronic state of the $\mathrm{Fe}$ (II) ion is a quintet while a singlet state is populated when $\mathrm{CO}$ is present. One of the difficulties about this approach is that, to first order, these states are not coupled. On the contrary, quintets and triplets as well as triplets and singlets, are coupled due to spin-orbit interactions. It is therefore more intuitive to think that dynamics might be nonadiabatic, and that crossings between triplets and quintets contribute to the population of the triplet states, which in turn give rise to transitions into the singlet ground state. Moreover, there is no reason to assume that only one quintet or triplet state is involved in this 

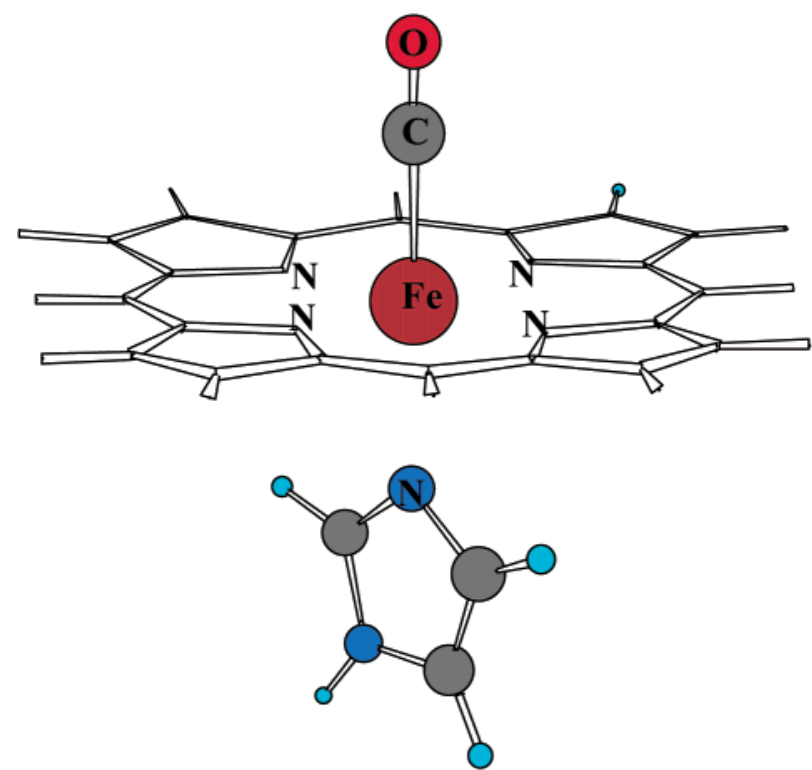

Figure 1. Snapshot of the molecular system used for ab initio calculations and for testing our semiempirical approach. It includes the heme ring, the $\mathrm{CO}$ molecule, the $\mathrm{Fe}(\mathrm{II})$ ion, and a histidine residue.

process. In the following sections we outline the strategy for the calculation of these coupled many-body potential energy surfaces.

\section{Methods}

We assume that the reader is somewhat familiar with the DIM approach so we will not go into much detail; however, for completeness we will outline the basic procedures for setting up the full system electronic Hamiltonian. Extensive examples, derivations, and applications can be found in the following articles and references therein. ${ }^{11-13,16-32}$ The DIM Hamiltonian is an exact rewriting of the full electronic Schroedinger Hamiltonian,

$$
\hat{H}=\sum_{i<j} \hat{H}_{i j}-(n-2) \sum_{i} \hat{H}_{i}
$$

where $\hat{H}_{i j}$ and $\hat{H}_{i}$ are diatomic and monatomic operators. They are defined so that they apply only to the electrons originally assigned to atom $i$ and atom $j$, respectively. For a rigorous description of how antisymmetrization is achieved, the best source is still the original papers by Ellison. ${ }^{11-13}$ The first summation runs over all possible pairs of atoms, while the second one runs over all atoms. The latter sum multiplied by $(n-2), n$ being the total number of atoms, is included to correct for the number of times monoatomic operators have been overcounted in the diatomic operator sum. In the semiempirical version of DIM, one starts with some basis set of the form

$$
\left|\phi_{1}, \phi_{2} \ldots \phi_{n}\right\rangle
$$

and usually approximates the diatomic matrix elements in the following way:

$$
\begin{array}{r}
\left\langle\psi_{1}, \psi_{2}, . . \psi_{n}\left|\hat{H}_{1,2}\right| \phi_{1}, \phi_{2} . . \phi_{n}\right\rangle=\left\langle\psi_{1}, \psi_{2}\left|\hat{H}_{1,2}\right| \phi_{1}, \phi_{2}\right\rangle \times \\
\left\langle\psi_{3}, . . \psi_{n} \mid \phi_{3}, . . \phi_{n}\right\rangle
\end{array}
$$

similarly for the monoatomic matrix elements

$$
\begin{aligned}
&\left\langle\psi_{1}, \psi_{2}, . . \psi_{n}\left|\hat{H}_{1}\right| \phi_{1}, \phi_{2} . . \phi_{n}\right\rangle=\left\langle\psi_{1}\left|\hat{H}_{1}\right| \phi_{1}\right\rangle \times \\
&\left\langle\psi_{2}, . . \psi_{n} \mid \phi_{2}, . . \phi_{n}\right\rangle
\end{aligned}
$$

In general, the atomic basis elements are assumed to be eigenstates of their corresponding atomic Hamiltonian, hence $\left\langle\psi_{1}\left|\hat{H}_{1}\right| \phi_{1}\right\rangle=\delta\left(\psi_{1}, \phi_{1}\right) \times E_{\phi_{1}}$. It is common practice to allow for electronic excitations only in a very small subset of atoms. This is justifiable in those cases when the excited states involved are localized in space. Depending on the choice of basis set elements, the DIM formalism allows for the study of both charge transfer and non-charge-transfer processes.

In particular, we are interested here in the case of transition metal ions surrounded by ligands that are, in principle, attached to some protein which in turn might be in solution. We do not wish to study at this point electronic processes that involve charge transfer to or from the ligands; hence, we are mainly concerned with the usual nonbonding or antibonding transitions that occur among $d$ orbitals of the transition metal ion in an arbitrary complex environment.

In general, DFT methods to calculate potential energy surfaces for transition metal ions with different spin multiplicities have proven to be very effective because of their accuracy and low computational cost. As already introduced, excited electronic states for each of these given multiplicities are hard to calculate, and SO coupling is usually not included. Ligand field theory as a semiempirical method has been widely applied in the past $^{33-37}$ and is still used in fitting and understanding electronic, Mössbauer, and Zeeman spectra of transition metal ions in proteins and other very complex environments, see for example refs 37 and 38 and references therein.

Within the same semiempirical spirit, we propose here to partition the DIM Hamiltonian in two: a first term involving all operators that include atom 1 (the metal ion), and a second term with the remaining operators.

$$
\begin{gathered}
\hat{H}=\hat{H}^{I}+\hat{H}^{I I} \\
\hat{H}^{I}=\sum_{i=1, j>i} \hat{H}_{i, j}-(n-2) \times \hat{H}_{1} \\
\hat{H}^{I I}=\sum_{i \geq 2, j>i} \hat{H}_{i, j}-(n-2) \times \sum_{i \geq 2} \hat{H}_{i}
\end{gathered}
$$

Within this approach we apply Gerloch's cellular AOM parametrization of the ligand field model as a means to compute an approximation to the matrix elements of $\hat{H}^{I}$. Only a few sites which are closest to the metal ion are assumed to be ligands. Interactions of the metal ion with atoms or molecules further away are assumed to be smaller and can be incorporated in some MM (molecular mechanics) fashion.

It is worthwhile noticing that if only one atom, i.e., the metal ion, is allowed to have electronic excitations, the basis elements of the space used will have the following fixed form:

$$
\left|\phi_{1}^{k}, \phi_{2} \ldots \phi_{n}\right\rangle
$$

where the subscript denotes atoms and the superscript the different excited electronic wave functions of the metal ion. All matrix elements of $\hat{H}^{I I}$, which of course do not involve atom 1 , the metal center, will be diagonal because of the approximation in eqs 3 and 4 .

$$
\begin{aligned}
&\left\langle\phi_{1}^{k}, \phi_{2} \ldots . \phi_{\mathrm{n}}\left|\hat{H}^{I I}\right| \phi_{1}^{k^{\prime}}, \phi_{2} \ldots . \phi_{\mathrm{n}}\right\rangle=\left\langle\phi_{1}^{k} \mid \phi_{1}^{k^{\prime}}\right\rangle \times \\
&\left\{\sum_{i \geq 2, j>i}\left\langle\phi_{i} \phi_{j}\left|\hat{H}_{i, j}\right| \phi_{i} \phi_{j}\right\rangle-(n-2) \times \sum_{i \geq 2}\left\langle\phi_{i}\left|\hat{H}_{i}\right| \phi_{i}\right\rangle\right\}
\end{aligned}
$$

All diagonal elements of the Hamiltonian will incorporate the same contribution from $\hat{H}^{I I}$. These contributions correspond 
to the ground-state Born-Oppenheimer (BO) energy surface for the interaction of atoms labeled 2 to $n$. In the usual semiempirical applications of DIM, these are assumed to be given by some force-field parametrization. In the following subsection we will describe how ligand field theory can be used to calculate at least in an approximate fashion, matrix elements of $\hat{H}^{I}$.

2.1. Angular Overlap Model. In this subsection we will briefly describe how an approximation to $\hat{H}^{I}$ is calculated. The ligand field Hamiltonian can be understood as a zero-th order perturbation over a set of unperturbed degenerate atomic states. ${ }^{39}$ After diagonalization of the perturbation Hamiltonian, gaps between different electronic states are obtained. Following Gerloch's approach, ${ }^{10}$ the ligand field Hamiltonian is written as

$$
\hat{H}_{\mathrm{LF}}=\sum_{i, j} U_{i, j}\left(r_{i, j}\right)+\sum_{i} V_{\mathrm{LF}}\left(R_{i}\right)+\xi \times \sum_{i} l_{i} \cdot s_{i}
$$

where $r_{i, j}$ is the distance between the $i$ th and $j$ th electrons, and $R_{i}$ is the $i$ th ligand to metal relative position. The first term corresponds to the electron-electron repulsion within the metal ion, the second is the ligand-metal interaction, and the third is the sum over the single electron spin-orbit coupling operators. The spin-orbit coupling operator is diagonal in the coupled $J$ representation, hence in what follows we will use the following basis set for the whole molecular system:

$$
\left|(J, M, L, S), \phi_{2}, . . \phi_{n}\right\rangle
$$

The quantum numbers in parentheses correspond to the coupled representation of the atomic electronic terms of the transition metal ion. We proceed writing, up to an additive constant, the DIM matrix elements in the following way:

$$
\sum_{k=2}^{n} \hat{H}_{1, k}-(n-2) \times \hat{H}_{1}=\hat{H}_{\mathrm{LF}}
$$

where in this case $n-1$ is the total number of atoms assumed to be ligands to the central ion. Recalling that we do not allow for electronic excitations on atoms other than the transition metal ion, the matrix elements for the electron-electron repulsion within the metal ion become

$$
\begin{aligned}
&\left\langle(J, M, L, S), \phi_{2}, . . \phi_{n}\left|\sum \frac{1}{r_{i, j}}\right|\left(J^{\prime}, M^{\prime}, L^{\prime}, S^{\prime}\right), \phi_{2}, . . \phi_{n}\right\rangle= \\
&\left\langle(J, M, L, S)\left|\frac{1}{r_{i, j}}\right|\left(J^{\prime}, M^{\prime}, L^{\prime}, S^{\prime}\right)\right\rangle
\end{aligned}
$$

Following Gerloch ${ }^{10}$ we write

$$
\begin{aligned}
& \left\langle(J, M, L, S)\left|\sum \frac{1}{r_{i, j}}\right|\left(J^{\prime}, M^{\prime}, L^{\prime}, S^{\prime}\right)\right\rangle= \\
& \sum_{k}\left\langle L, S|| F^{k}|| L^{\prime}, S^{\prime}\right\rangle F^{k} \delta\left(L, L^{\prime}\right) \times \delta\left(S, S^{\prime}\right) \times \\
& \delta\left(J, J^{\prime}\right) \times \delta\left(M, M^{\prime}\right)
\end{aligned}
$$

where the reduced matrix elements $\left\langle L, S|| F^{k}|| L^{\prime}, S^{\prime}\right\rangle$ are tabulated in Nielson and Koster, ${ }^{40}$ and $F^{k}$ are the Condon-Shortley radial integrals. Spin-orbit coupling matrix elements are calculated in a similar way: ${ }^{10}$

$$
\begin{aligned}
& \left\langle(J, M, L, S), \phi_{2}, . . \phi_{\mathrm{n}}\left|\sum l_{i} \cdot s_{i}\right|\left(J^{\prime}, M^{\prime}, L^{\prime}, S^{\prime}\right), \phi_{2}, . . \phi_{n}\right\rangle= \\
& \left\langle(J, M, L, S)\left|\sum l_{i} \cdot s_{i}\right|\left(J^{\prime}, M^{\prime}, L^{\prime}, S^{\prime}\right)\right\rangle=\left\langle L, S|| V^{11}|| L^{\prime}, S^{\prime}\right\rangle \times \\
& \delta\left(J, J^{\prime}\right) \times \delta\left(M, M^{\prime}\right) \times(-1)^{L^{\prime}+S+J} \times(l(l+1)(2 l+1))^{1 / 2} \times \\
& \left\{\begin{array}{lll}
L & S & J \\
S^{\prime} & L^{\prime} & 1
\end{array}\right\}
\end{aligned}
$$

The double tensor reduced unit matrices $\left\langle L, S|| V^{11}|| L^{\prime}, S^{\prime}\right\rangle$ are tabulated in Nielson and $\operatorname{Koster}^{40}$ as well. Finally we are left with the calculation of the ligand-metal interactions:

$$
\left\langle(J, M, L, S), \phi_{2}, . . \phi_{n}\left|\sum_{i} V_{\mathrm{LF}}\right|\left(J^{\prime}, M^{\prime}, L^{\prime}, S^{\prime}\right), \phi_{2}, . . \phi_{n}\right\rangle
$$

In the cellular version of $\mathrm{AOM},{ }^{10}$ the sum over ligands $\sum_{i} V_{\mathrm{LF}}$ is expanded in terms of spherical harmonics operators. Only matrix elements of $Y_{0}^{0}, Y_{q}^{2}$, and $Y_{q}^{4}$ survive due to the fact that the basis set for the metal ion includes only $\mathrm{d}$ orbitals:

$$
\begin{aligned}
& \left\langle(J, M, L, S), \phi_{2}, . . \phi_{n}\left|\sum_{i} V_{\mathrm{LF}}\right|\left(J^{\prime}, M^{\prime}, L^{\prime}, S^{\prime}\right), \phi_{2}, . . \phi_{n}\right\rangle= \\
& \sum_{k} \sum_{q=-k}^{k} c_{k q}(R)\left\langle(J, M, L, S), \phi_{2}, . . \phi_{n}\left|Y_{q}^{k}\right|\left(J^{\prime}, M^{\prime}, L^{\prime}, S^{\prime}\right), \phi_{2}, . . \phi_{n}\right\rangle
\end{aligned}
$$

Here $R$ is a multidimensional vector representing the relative coordinates of all the ligands to the metal ion. The $c_{k q}(R)$ spherical tensor global coefficients can be cast in terms of the properly rotated local frame $\sigma, \pi$, and $\delta$ energies (see Table 9.1 in reference 10$)$.

$$
\begin{aligned}
\left\langle d_{z^{2}}\left|v^{1}\right| d_{z^{2}}\right\rangle & =e_{\sigma}^{l} \\
\left\langle d_{x z}\left|v^{l}\right| d_{x z}\right\rangle & =e_{\pi x}^{l} \\
\left\langle d_{y z}\left|v^{l}\right| d_{y z}\right\rangle & =e_{\pi y}^{l} \\
\left\langle d_{x y}\left|v^{l}\right| d_{x y}\right\rangle & =e_{\delta x y}^{l} \\
\left\langle d_{x^{2}-y^{2}}\left|v^{1}\right| d_{x^{2}-y^{2}}\right\rangle & =e_{\delta x^{2}-y^{2}}^{l}
\end{aligned}
$$

For a detailed description of the way in which the expansion in terms of $Y_{q}^{k}$ operators is inverted to obtain the relations between the local or global frame spherical tensor coefficients $c_{k q}$ in terms of $\sigma, \pi$, and $\delta$ energies, the reader is advised to look at the original derivations by Gerloch ${ }^{34}$ and the book by Silver. ${ }^{41}$ Much of the transition metal chemistry literature is devoted to finding the proper value of these interactions for different ligands at their equilibrium distance from the metal ion. Linear combinations of these correspond to the usual 10 $D_{q}$ splitting in octahedral and tetrahedral complexes. ${ }^{10,39}$ Interactions $\sigma, \pi$, and $\delta$ are diagonal in a local frame of reference that has each particular ligand-ion bond in the local $Z$ axis. To correctly add up the different ligand contributions, these local diagonal Hamiltonians must be rotated using the Wigner rotation matrices to the lab frame in which the total Hamiltonian is written. ${ }^{10,30,31,34}$ On performing these unitary transformations for each of the ligands, the ligand field Hamiltonian matrix becomes in the most general case off-diagonal and complex. In eq $15, Y_{0}^{0}$ is a scalar operator, therefore its matrix elements are some constant times the unit matrix and do not contribute to the gaps between electronic states. This is why in the usual 
cellular parametrization $c_{00}$ is set to zero. It is important to note that $c_{00}$ is in fact $c_{00}(R)$. In our parametrization scheme, we leave $c_{00}(R)$ out for the sake of fitting the gaps between electronic states (this is achieved by fitting $\sigma, \pi$, and $\delta$ to an appropriate functional form), but use it as an extra degree of freedom to fit the singlet ground-state energy function. Matrix elements of the spherical harmonics are calculated ${ }^{10}$ in the following way:

$$
\begin{gathered}
\left\langle(J, M, L, S), \phi_{2}, . . \phi_{n}\left|Y_{q}^{k}\right|\left(J^{\prime}, M^{\prime}, L^{\prime}, S^{\prime}\right), \phi_{2}, . . \phi_{\mathrm{n}}\right\rangle= \\
(-1)^{J+J^{\prime}+L+S+M+k+1} \times\left((2 J+1)\left(2 J^{\prime}+1\right)\right)^{1 / 2} \times(2 l+1) \times \\
((2 k+1) /(4 \pi))^{1 / 2} \times\left(\begin{array}{lll}
l & k & l \\
0 & 0 & 0
\end{array}\right) \times\left(\begin{array}{lll}
J & k & J^{\prime} \\
-M & q & M^{\prime}
\end{array}\right) \times \\
\left\{\begin{array}{lll}
L & J & S \\
J^{\prime} & L^{\prime} & k
\end{array}\right\} \times\left\langle L, S|| U^{k}|| L^{\prime}, S\right\rangle \times \delta\left(S, S^{\prime}\right)
\end{gathered}
$$

where the unit tensor matrix elements $\left\langle L, S|| U^{k}|| L^{\prime}, S\right\rangle$ are obtained from Nielson and Koster. ${ }^{40}$

\section{Parametrization Scheme}

In the previous section we showed how, at least in a semiempirical fashion, one is able to cast DIM in terms of force field potentials and ligand field parameters. The force field potentials for the ligand-ligand interactions and for species far from the metal center are widely available. They enter the DIM Hamiltonian matrix in a very simple diagonal form that corresponds to the interaction of species on their ground-state Born-Oppenheimer potential energy surface. The necessary parameters to fit the metal ligand interactions, namely one $\sigma$, two $\pi$, and two $\delta$ local energies for each ligand, are to be obtained either from fits to ab initio calculations or in the more conventional way to spectra. The problem arising from fitting to spectra is that one cannot, in general, extract the $R$ dependency of the parameters. Ab initio calculations can provide detailed information about the ground state of each spin multiplicity in a particular system as a function of ligand coordinates. This information, however, may not be enough to obtain the functional form of the ligand field parameters, it is known that in the absence of the magnetic interaction the same "correct" eigenvalues can be obtained with more than one combination of $\sigma$ and $\pi$ local energies, hence some criteria has to be used to deal with this over-parametrization of the model. ${ }^{10,39}$

In the method we present here, the eigenvalues of the whole system Hamiltonian are a function of the aforementioned $\sigma$ $\left(R_{i}\right), \pi\left(R_{i}\right)$, and $\delta\left(R_{i}\right)$ local energy functions. To reduce overparametrization, some simplification in parameter space should be implemented. ${ }^{10,39}$ Being much smaller than $\sigma\left(R_{i}\right)$ and $\pi\left(R_{i}\right)$ energies, $\delta\left(R_{i}\right)$ interactions are usually dropped from the computation. For the particular implementation of $\mathrm{CO}$ in myoglobin, further restrictions can be applied. The CO molecule has cylindrical symmetry, hence both $\pi\left(R_{i}\right)$ interactions, between $\mathrm{CO}$ and the metal ion, should be assigned the same value. Furthermore the heme ring is assumed to be a four-ligand system; each of the ligands being the same should possess the same set of $\sigma\left(R_{i}\right)$ and $\pi\left(R_{i}\right)$ energy functions. In this case, however, as opposed to the case of $\mathrm{CO}, \pi_{x}\left(R_{i}\right)$, and $\pi_{y}\left(R_{i}\right)$ are not equivalent because of the lack of symmetry. The same argument holds for the sixth ligand, a histidine residue. Hence the total number of functions to be fitted in this particular application is eight.

As we should recall, ligand field theory provides us with the corresponding splittings between otherwise degenerate electronic states. However, to obtain absolute energies we still need one more parametrization step. We use the extra degree of freedom $c_{00}(R)$ to fit the absolute value of the singlet ground state of the system. Having obtained the gaps, this fixes the absolute value of the energy eigenvalues of the system.

For the particular application we present in the following section, we did not fit the ground state singlet energy, we simply used the ab initio calculated values and added the gaps obtained from DIMSELF to obtain the excited-state potential energy surfaces.

\section{Results}

We generated a set of eighteen geometries for the reduced system displayed in Figure 1 composed of the heme ring, the $\mathrm{Fe}(\mathrm{II})$ ion, the $\mathrm{CO}$ molecule, and a histidine residue. ${ }^{42}$ The configurations were chosen along different cuts in the potential energy landscape of our reduced system. In each of them, the $\mathrm{CO}$ molecule was kept at a fixed angle with the $\mathrm{C}$ atom pointing toward the $\mathrm{Fe}(\mathrm{II})$ ion and pulled upward perpendicular to the heme ring. The difference between these curves is that in each case the remaining atoms were allowed to relax, assuming the system was either in the ground singlet, triplet, or quintet state. Hence we obtained nine ab initio potential energy curves. A singlet, a triplet, and a quintet, with geometries minimized according to the singlet are displayed in Figure 2a; a singlet, a triplet, and a quintet minimized for the triplet are displayed in Figure 2b; and, finally, a singlet, a triplet, and a quintet minimized for the quintet are depicted in Figure 2c. One interesting feature about these ab initio curves is the fact that neither the ground state triplet nor the ground state quintet cross the ground state singlet in Figure 2a, while they do in the case of Figure $2 b$ and $c$. In Figure $2 b$ the ground state triplet is lower in energy than the quintet, while in Figure $2 c$ the lower one is the quintet. This clearly points to the fact that conical intersections between these two exist, and spin-orbit coupling interactions could lead to nonadiabatic transitions.

The first stringent test our proposed method should pass is being capable of reproducing the ab initio energy functions in Figure 2. Figure 3 presents the same energy plots as in Figure 2 computed with our DIMSELF method. The solid lines correspond to the DIMSELF surfaces, while the symbols correspond to the ab initio values. As can be seen the agreement is excellent. In Figure 3a there seems to be a discontinuity in the ground-state triplet at short values of the $\mathrm{CO}-\mathrm{Fe}$ (II) distance. This prediction of DIMSELF actually corresponds to an excited triplet state crossing that seems to occur at around $1.8 \AA$. This crossing may explain the apparently different results reported in two papers recently published in the literature. ${ }^{14,15}$

In a recent work, using the same model system we employ here, Harvey ${ }^{15}$ computed three minimum energy crossing point geometries using a DFT method for the singlet to quintet, singlet to triplet, and triplet to quintet ground states. With these and the energy at infinite separation of the $\mathrm{CO}$ from the ring, he is able to estimate energy barriers for "adiabatic transitions" between them. It is clear from Figure 1 in Harvey's work ${ }^{15}$ that both triplet and quintet ground states are dissociative curves. Other recent work by McMahon and co-workers ${ }^{14}$ seems to indicate that the triplet ground state is actually bound, the minimum of this triplet state being at a $\mathrm{CO}-\mathrm{Fe}$ (II) distance of about $1.8 \AA$ A. In Figure 4, we show the ground-state singlet and first few excited triplet and quintet states computed using DIMSELF. Geometries are chosen to be the same as those employed in our ab initio calculations, and for the sake of comparison with them, the effect of SO coupling was left out of the plots.

In Figure $4 \mathrm{a}$ we observe that although the adiabatic groundstate triplet seems to be dissociative, it is in fact composed of 


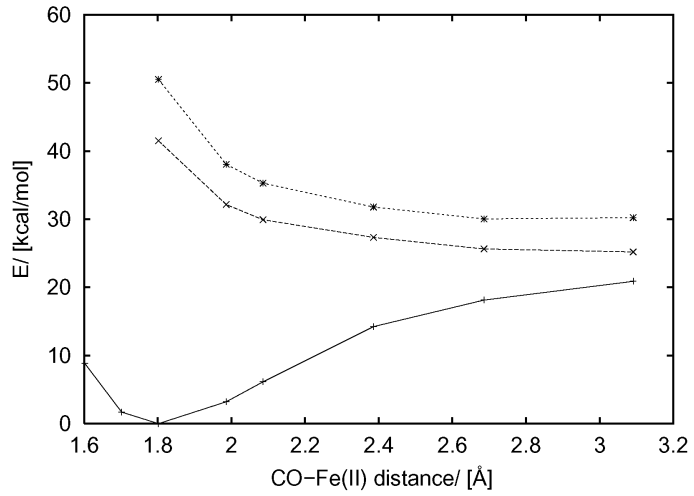

(a)

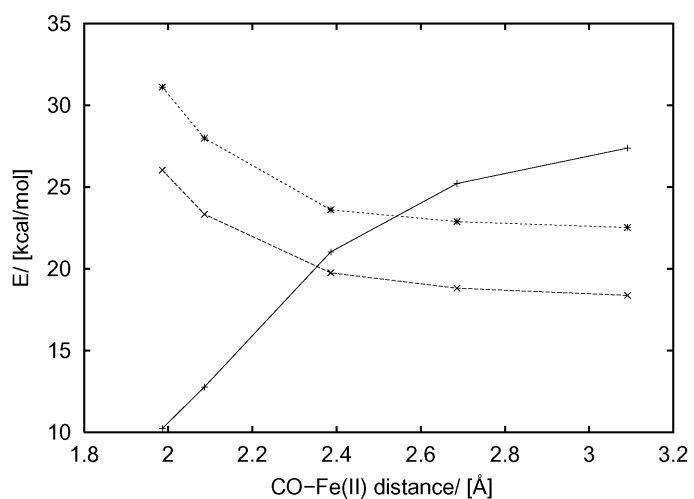

(b)

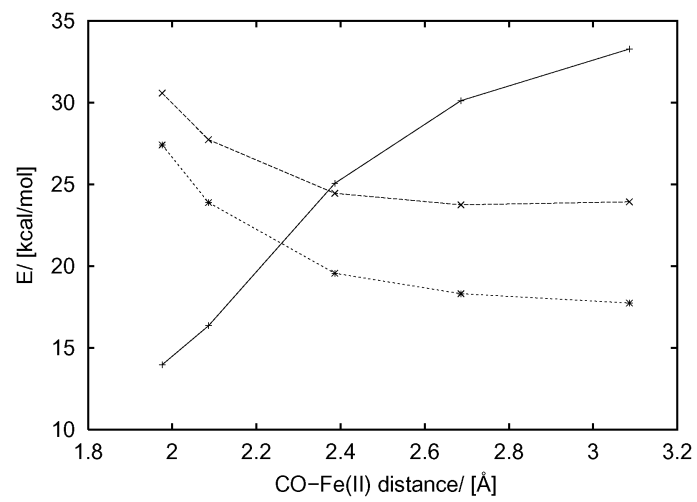

(c)

Figure 2. (a), (b), and (c) display ab initio potential energy scans of the $\mathrm{CO}-\mathrm{Fe}$ distance perpendicular to the heme ring minimized according to ground-state singlet, triplet and quintet, respectively. Solid lines corresponds to ground-state singlet while dashes and points correspond to ground-state triplet and quintet, respectively.

at least two crossing diabats. Our DIMSELF results indicate that the aforementioned discrepancies between the two papers is the result of following a symmetry state and not an energy eigenstate. To further test the validity of this conclusion, we have computed ${ }^{43}$ single-point time-dependent density functional excited-state energies (TDDFT) ${ }^{44,45}$ at several geometries other than those depicted in Figure 4a. Time-dependent density functional methods have proven to be very useful in the calculation and interpretation of spectra of transition metal compounds. Comparison of the accuracy of the TDDFT results in the case of transition metal ions against other ab initio methods and against experiments can be found in refs 46-48 and references therein. In all cases, TDDFT compares well with other more expensive fully correlated ab initio methods. In fact, it seems to produce better results than most other methods in

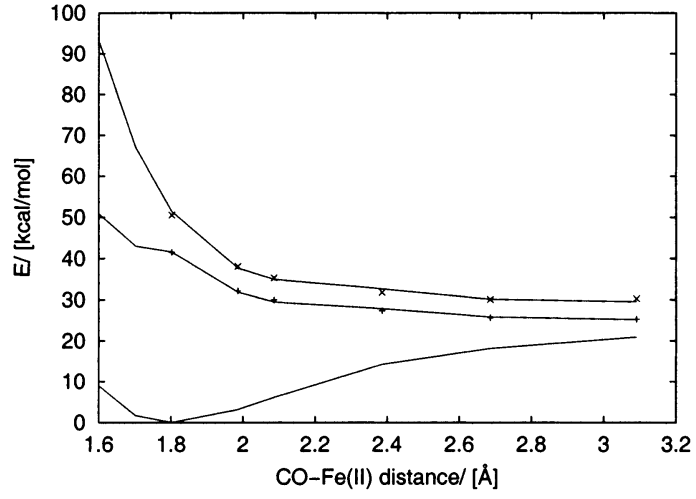

(a)

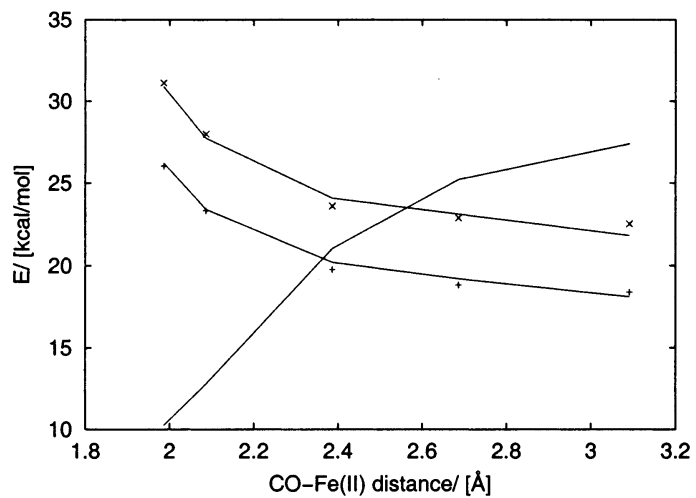

(b)

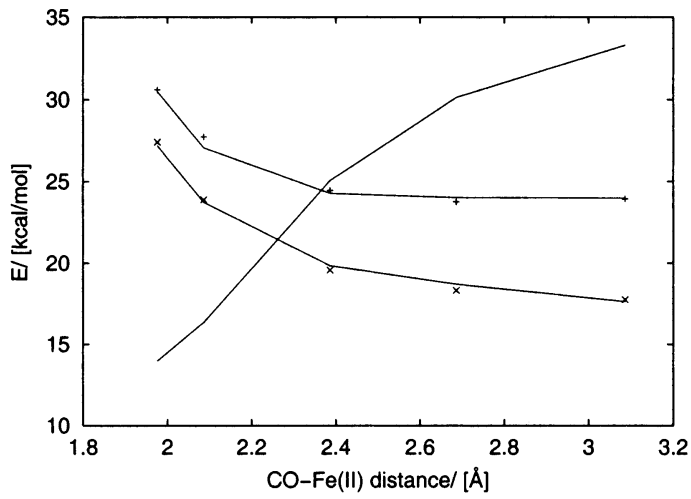

(c)

Figure 3. Parts (a), (b), and (c) display with solid lines, potential energy scans minimized according to ground state singlet, triplet and quintet respectively using our semiempirical method. Overlayed on the same graphs with plus signs and crosses are the ab initio results for the triplet and quintet, respectively.

the cases studied by Gisbergen ${ }^{46}$ and co-workers. The accuracy of these methods ${ }^{46}$ is on the order of a few tenths of an eV compared to experiments. Figure 5a shows the singlet ground state and the first three triplet states as obtained with TDDFT. Figure $5 \mathrm{~b}$ shows the same results obtained with our DIMSELF method. The agreement between our DIMSELF approach and TDDFT is quite remarkable if we consider the fact that no excited-state information was used to fit the energy functions deployed in the DIMSELF calculation. Tables 1 and 2 display a comparison of the gaps we obtained between triplet states and the ground-state singlet using DIMSELF and TDDFT. The TDDFT calculations predict a crossing between triplet states at about $1.8 \AA$, and another crossing at $2 \AA$. This is exactly the same pattern we observe in Figure $5 \mathrm{~b}$ and in Tables 1 and 2. Preliminary results from our group also confirm the existence of crossings between quintet states as predicted by our DIM- 


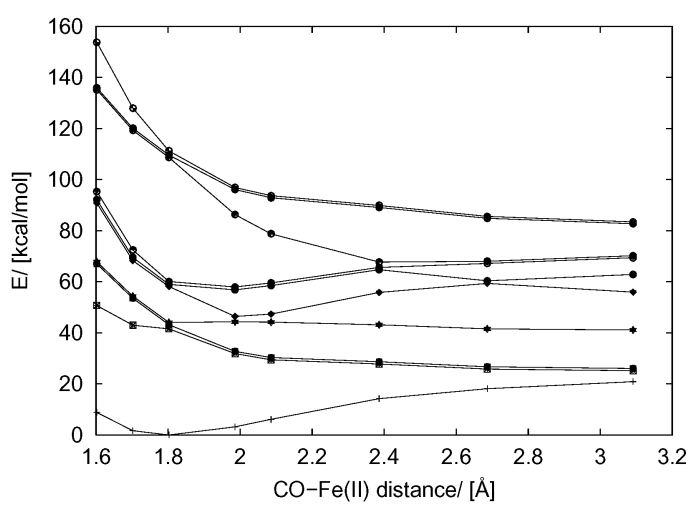

(a)

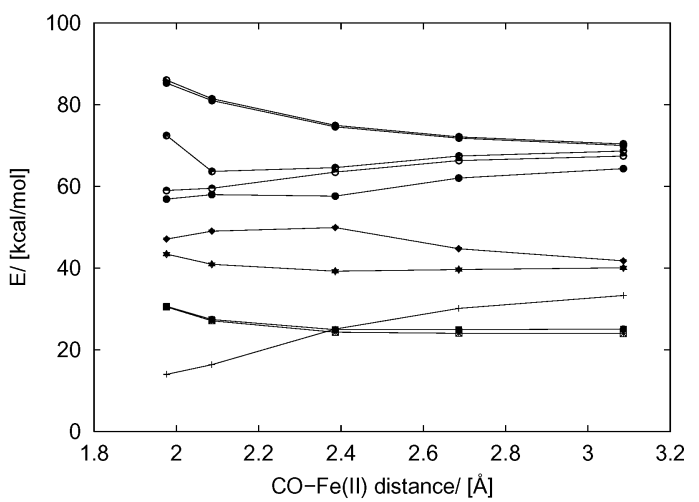

(c)

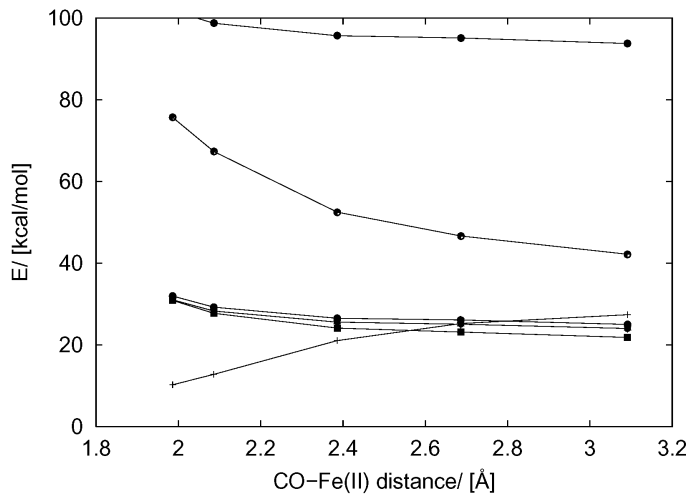

(e)

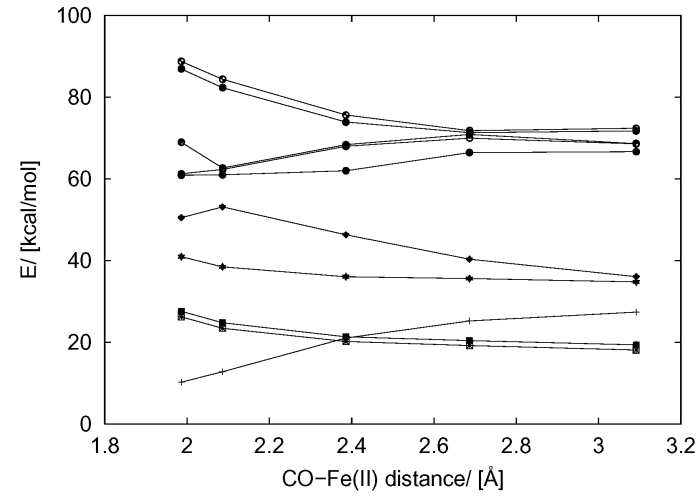

(b)

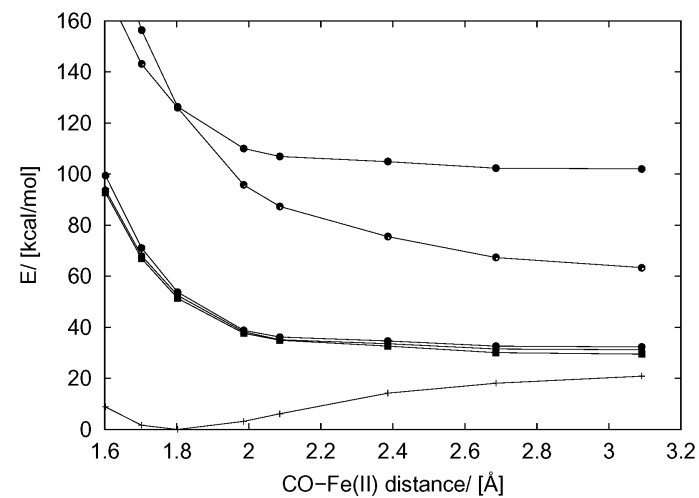

(d)

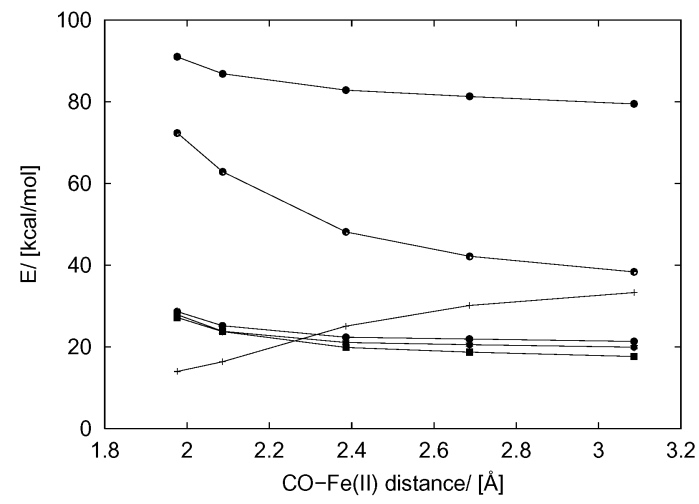

(f)

Figure 4. Parts (a)-(c) correspond to ground-state singlet, ground-state triplet, and first few excited triplet states computed with our semiempirical approach. Part (a) corresponds to geometries minimized for the ground singlet state, while (b) and (c) correspond to geometries minimized for the triplet and quintet ground state, respectively. The geometries are the same as those used in Figure 2. Parts (d) - (f) show analogous curves only in this case ground-state singlet, ground-state quintet, and first few excited quintet states are displayed. It is important to notice that solid lines follow "adiabatic" potential energy surfaces as opposed to "diabatic" states, hence the lack of smoothness around crossing points.

SELF method. Our preliminary data indicate that DIMSELF predicts the correct first excited singlet state energy, at least qualitatively as well.

Another interesting feature of the scans displayed in Figure 4 is the fact that there are excited quintet and triplet states that cross the ground singlet state as well. As we mentioned earlier for different geometries, these manifolds of states actually change in energy order. In some cases the triplets are lower than the quintets, while in other cases the opposite is true. This can be better appreciated in Figure 6 where we plot groundstate singlet and the first few quintets and triplets on the same graph. Harvey ${ }^{15}$ predicts a barrier for crossings from quintet ground state to the singlet ground state, while McMahon's results seem to indicate that the crossing occurs without a barrier. The question arises as to whether these differences are significant or not: Are these barriers important for the actual dynamics? Is it possible to estimate transition rate constants from them? From our results, it is clear that the picture presented in refs 14 and 15 is incomplete. Dynamics in this system will involve the crossing of many fluctuating barriers and different transition states. Given the fact that several quintets and triplets cross, there is no a priori reason to assume that the dynamics would be completely adiabatic, or completely diabatic. Furthermore, there is no reason to assume that only the ground triplet or quintet state is involved in the dynamics. Our quantum mechanics-molecular mechanics (QMMM) calculations currently in progress, on the whole protein, performed using the $\mathrm{JAGUAR}^{42}$ package point to the fact that in some cases the order of the quintet and triplet ground state is reversed from that obtained in the reduced system.

As we mentioned before, when the $\mathrm{CO}$ molecule is confined in the pocket, the environment will greatly affect the "gas-phase 


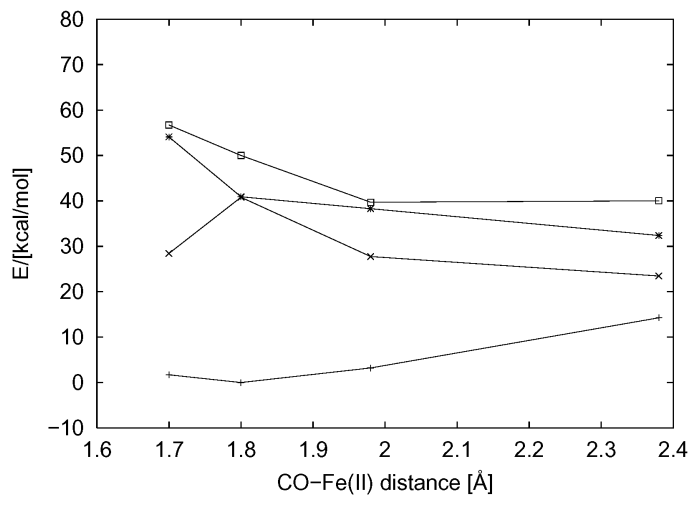

(a)

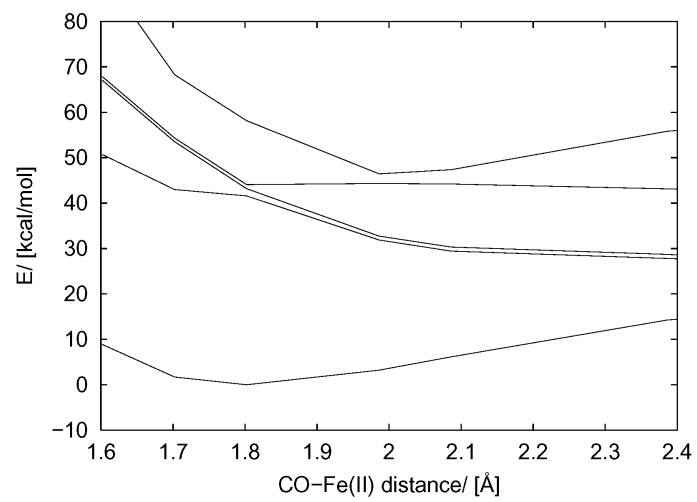

(b)

Figure 5. Part (a) corresponds to the singlet ground state and the first three triplet states computed with excited-state density functional theory at four significant geometries minimized for the singlet ground state. Part (b) shows the same energy levels computed with DIMSELF.

TABLE 1: DIMSELF Triplet to Singlet Gaps in $\mathrm{eV}^{a}$

\begin{tabular}{cccc}
\hline $\begin{array}{r}\mathrm{CO}-\mathrm{Fe}(\mathrm{II}) \\
\text { distance in } \AA\end{array}$ & lowest triplet & first excited & second excited \\
\hline 1.7 & 1.79 & 2.27 & 2.87 \\
1.8 & 1.82 & 1.91 & 2.52 \\
1.98 & 1.25 & 1.77 & 1.86 \\
2.38 & 0.6 & 1.25 & 1.81
\end{tabular}

${ }^{a}$ We only consider gaps between states that are nondegenerate. Each of the triplet states is in fact either degenerate or quasi-degenerate.

TABLE 2: TDDFT Triplet to Singlet Gaps in $\mathrm{eV}$

\begin{tabular}{cccc}
\hline $\begin{array}{r}\mathrm{CO}-\mathrm{Fe}(\mathrm{II}) \\
\text { distance in } \AA\end{array}$ & lowest triplet & first excited & second excited \\
\hline 1.7 & 1.16 & 2.27 & 2.39 \\
1.8 & 1.77 & 1.77 & 2.17 \\
1.98 & 1.06 & 1.52 & 1.58 \\
2.38 & 0.41 & 0.80 & 1.13
\end{tabular}

looking" potential energy curves depicted in Figure 2. Hence even disregarding all possible nonadiabatic channels through excited triplet and quintet states, it is very difficult to obtain dynamical information such as rate constants for these kind of complex systems from "gas-phase-like" models. In Figure 7 we present an example of this. As in all previous plots we displace the $\mathrm{CO}$ molecule in the direction perpendicular to the heme plane. In this case, however, using the DIM formalism we include part of the pocket structure. We find as expected a large repulsion barrier corresponding to the $\mathrm{CO}$ molecule colliding with the protein. Different conformations of the protein will accommodate different geometries of the $\mathrm{CO}$ molecule. Barriers for crossings between electronic states will fluctuate accordingly. Hence we strongly believe that a study of dynamics including

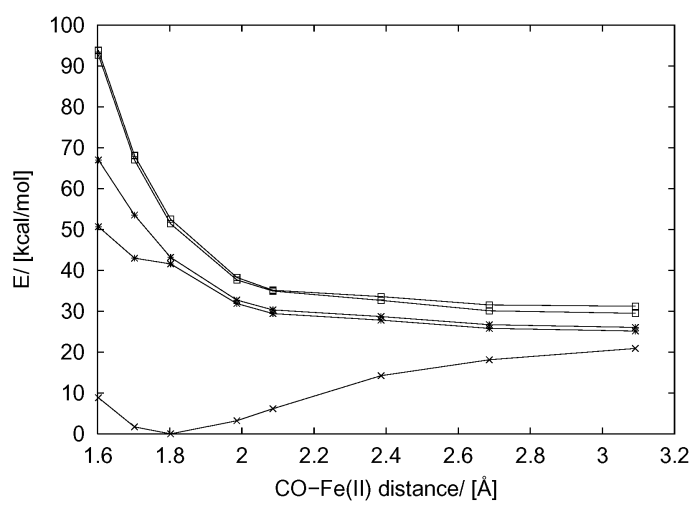

(a)

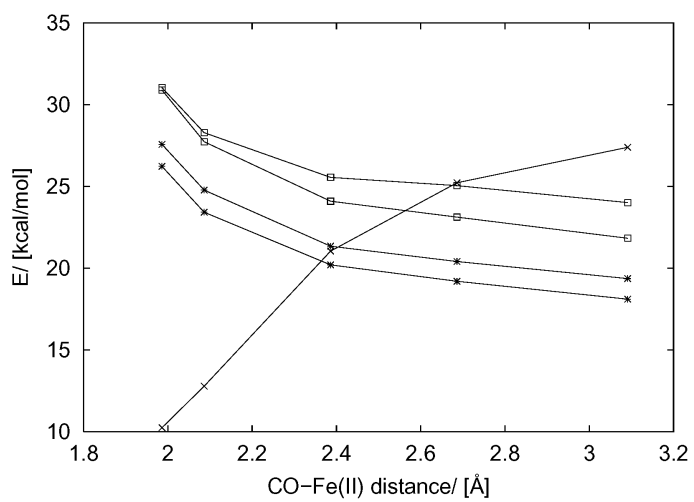

(b)

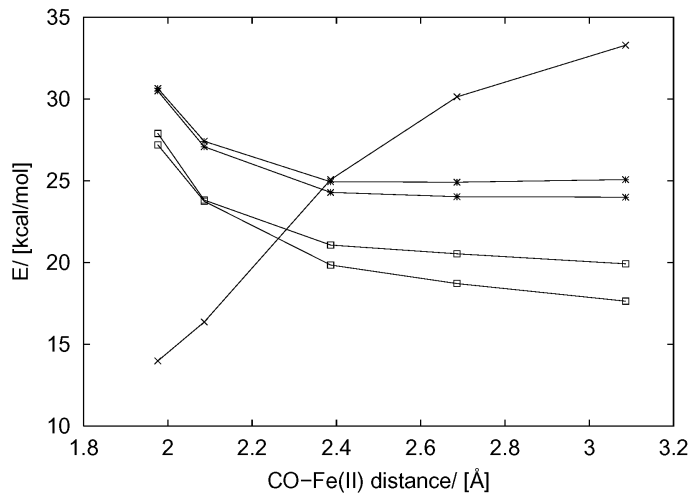

(c)

Figure 6. Parts (a) - (c) display, in the same graph, the ground-state singlet (lowest in energy at $2 \AA$ ) and first few triplet and quintet states. In parts (a) and (b), triplets are lower in energy than quintets, while in part (c) quintets are lower than triplets.

the protein is important to really shed light on the nature of the processes involved in these kind of chemical reactions.

\section{Conclusions}

In this paper, we have shown how ligand field theory and diatomics in molecules can be connected to generate ground and excited electronic potential energy surfaces of complex systems with transition metal ions. The ligand-ion potentials calculated using the AOM model are cast in terms of the usual DIM monatomic and diatomic matrix elements, the ligandligand and other nonmetal interactions are calculated as usual in the semiempirical DIM approach assuming they enter as diagonal contributions in the electronic Hamiltonian. The computational cost of adding extra atoms not considered "ligands" to the metal ion is the same as in MM calculations.

To perform dynamical studies, more configurations will need to be added to better fit $\sigma$ and $\pi$ energy functions. One could 


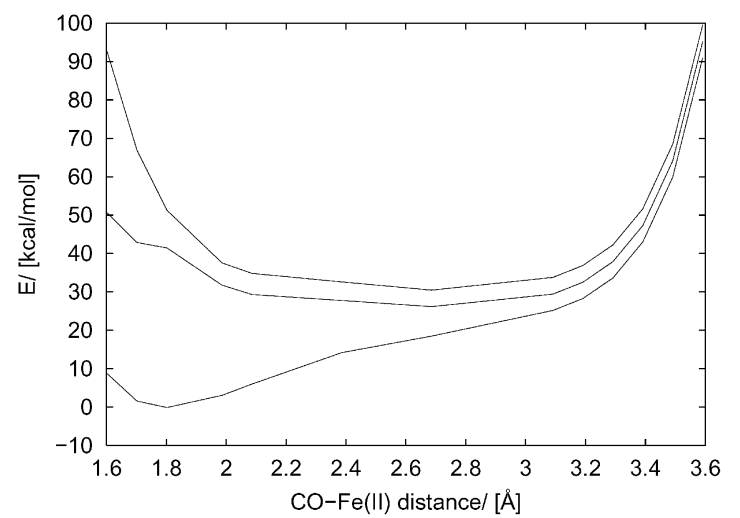

Figure 7. Ground-state singlet, triplet, and quintet computed for geometries minimized for the ground-state singlet. In this case using DIM we included the repulsive effect of the presence of the pocket amino acids.

also use the excited-state information that TDDFT provides to better adjust the energy of the excited-state potentials in an iterative fashion.

In our calculations, parameters were not fit to reproduce excited states; however, they do predict the correct energy structure for them. This result is very exciting. Although further testing on other systems must be performed to establish the transferability of these results, current data suggests that this opens the possibility for inexpensive study of excited-state reaction dynamics, light induced phenomena, and would offer a general method for computing excited states, that is both accurate and computationally feasible.

From a computational point of view, once parameters are known, this scheme is simple and fast (fractions of a second on a desktop PC) allowing it to be applied many times on-thefly. Moreover, it includes the necessary couplings due to spinorbit and configuration interaction, and hence correctly describes most of the otherwise forbidden transitions in the chemistry of transition metal ions in complex systems. However, states that correspond to charge transfer from the metal ion to the ligands or from the ligands to the metal ions as well as excited electronic states of the ligands themselves will not be captured with the current choice of basis set elements. These states could be important in the case where excitation energies to them are similar to the corresponding d energy splitting in the metal ion.

We believe that the semiempirical information about excited states provided here for the myoglobin system will be very useful to spectroscopists and ab initio quantum chemists in search of the exact characterization of avoided crossings and conical intersections. In this case in particular, it helped us understand the seemingly contradictory data available in the literature and the discontinuities in our own ab initio groundstate triplet potential energy curve. Without the evidence we obtained from our DIMSELF method, one could have believed that those discontinuities were due to poor convergence in the $\mathrm{ab}$ initio calculations.

Even in those cases where this method might not provide accurate quantitative information, it will at least provide qualitative information about excited states cheaply and thus can be used to target those molecular configurations expected to be important for avoided crossings and transition states.

We plan in the future, to extend our ab initio computations to better fit other regions of configuration space. With this we will recalculate our energy functions to make them reliable over a larger region of space. We also plan to add the rest of the protein and solvent, which should increase the computational cost only in the same way it does in a molecular mechanics calculation. Finally we plan to compare these results with more expensive QMMM calculations, specially in regions of interest where avoided crossings occur.

Acknowledgment. We gratefully acknowledge the generous allocation of computer time at the Boston University Super Computer Center. R.A.F. acknowledges support from the NIH (GM-40526). B.J.B. acknowledges support from the NIH (GM43340). V.G. acknowledges the Spanish Ministry for a postdoctoral fellowship.

\section{References and Notes}

(1) Frauenfelder, H.; Wolynes, P. G. Science 1985, 229, 337. 251 .

(2) Hanggi, P.; Talkner, P.; Borkovec, M. Rev. Mod. Phys. 1990, 62,

(3) Zheng, C.; Makarov, V.; Wolynes, P. G. J. Am. Chem. Soc. 1996, $118,2818$.

(4) Eaton, W. A.; Hanson, L. K.; Stephens, P. J.; Sutherland, J. C.; Dunn, J. B. R. J. Am. Chem. Soc. 1978, 100, 4991.

(5) Case, D. A.; Huynh, B. H.; Karplus, M. J. Am. Chem. Soc. 1979, $101,4433$.

(6) Rovira, C.; Kunc, K.; Hutter, J.; Ballone, P.; Parrinello, M. J. Phys. Chem. A 1997, 101, 8914. 247.

(7) Rovira, C.; Ballone, P.; Parrinello, M. Chem. Phys. Lett. 1997, 271

(8) Straub, J. E.; Karplus, M. Chem. Phys. 1991, 158, 221. 7063.

(9) Sagnella, D. E.; Straub, J. E. J. Phys. Chem. B 2001, 105, 7057-

(10) Gerloch, M. Magnetism and Ligand-Field Analysis; Cambridge University Press: Cambridge, 1983.

(11) Ellison, F. J. Am. Chem. Soc. 1963, 85, 3540.

(12) Ellison, F.; Huff, N.; Patel, J. J. Am. Chem. Soc. 1963, 85, 3544.

(13) Ellison, F.; Patel, J. J. Am. Chem. Soc. 1964, 86, 2115.

(14) McMahon, B. H.; Stojkovic, B. P.; Hay, P. J.; Martin, R. L.; Garcia,

A. E. J. Chem. Phys. 2000, 113, 6831.

(15) Harvey, J. N. J. Am. Chem. Soc. 2000, 122, 12401.

(16) Last, I.; George, T. J. Chem. Phys. 1987, 86, 3787.

(17) Last, I.; George, T. J. Chem. Phys. 1987, 87, 1183.

(18) Huestis, D.; Schlotter, N. J. Chem. Phys. 1969, 69, 3100

(19) Buchachenko, A.; Stepanov, N. J. Chem. Phys. 1996, 104, 9913.

(20) Grigorenko, B.; Nemukhin, A.; Apkarian, V. J. Chem. Phys. 1996, 104,5510 .

(21) Kuntz, P. J. The Diatomics-in-Molecules Method and the Chemical Bond; Maksic, Z. B., Ed.; Springer-Verlag: Berlin, 1990.

(22) Vongrunberg, H.; Gersonde, I.; Gabriel, H. Zeit. Fur Phys. D 1993, 28,145 .

(23) Tully, J. J. Chem. Phys. 1973, 59, 5122

(24) Gersonde, I.; Gabriel, H. J. Chem. Phys. 1993, 98, 2094.

(25) Krylov, A.; Gerber, R.; Apkarian, V. Phys. Chem. 1994, 189, 261.

(26) Krylov, A.; Gerber, R. Chem. Phys. Lett. 1994, 231, 395.

(27) Batista, V. S.; Coker, D. F. J. Chem. Phys. 1996, 105, 4033.

(28) Margulis, C. J.; Coker, D. F. J. Chem. Phys. 1999, 110, 5677.

(29) Margulis, C. J.; Horner, D.; Bonella, S.; Coker, D. F. J. Phys. Chem. A 1999, 103, 9552.

(30) Margulis, C. J.; Coker, D. F. J. Chem. Phys. 2000, 113, 6113.

(31) Margulis, C. J.; Coker, D. F. J. Chem. Phys. 2001, 114, 6744.

(32) Batista, V. S.; Coker, D. F. J. Chem. Phys. 1997, 106, 6923.

(33) Schaffer, C. E. Struct. Bonding 1968, 5, 68.

(34) Gerloch, M.; McMeeking, R. F. J. Chem. Soc., Dalton Trans. 1975, 2443.

(35) Stevens, K. W. H. Proc. R. Soc. London., A 1953, 219, 542.

(36) Bencini, A.; Benelli, C.; Gatteschi, D. Coord. Chem. Rev. 1984, $60,131$.

(37) Bencini, A.; Ciofini, I.; Uytterhoeven, M. G. Inorg. Chim. Acta 1998, 274, 90.

(38) Schunemann, V.; Winkler, H. Rep. Prog. Phys. 2000, 63, 263353.

(39) Figgis, B. N.; Hitchman, M. A. Ligand Field Theory and its Applications; Wiley-VCH: New York, 2000.

(40) Nielson, C. W.; Koster, G. F. Spectroscopic Coefficients for the $p^{\mathrm{n}}, d^{\mathrm{n}}$, and $f^{\mathrm{n}}$ Configurations; The M. I. T. Press: Cambridge, Massachusetts, 1963.

(41) Silver, B. L. Irreducible Tensor Methods; Academic Press: New York, 1976.

(42) All ground-state minimizations and single point calculations were performed with JAGUAR using restricted open density functional theory with B3LYP functional. The model employed is depicted in Figure 1. The basis set used is 6-31G* for all the atoms but Lacvp** for Fe, and 6-31G** 
for the $\mathrm{CO}$ and $\mathrm{N}$ bond to the iron. JAGUAR 4.1, Schrödinger, Inc., Portland, OR, 1991-2000.

(43) All excited-state TDDFT single-point calculations were performed with Q-Chem 2.0 using the B3LYP functional. The model employed is depicted in Figure 1. The basis set used is $6-31 \mathrm{G}^{*}$ for all the atoms but Lan2dz for Fe. Q-Chem 2.0: A high-performance ab initio electronic structure program, Kong, J.; White, C. A.; Krylov, A. I.; Sherrill, C. D.; Adamson, R. D.; Furlani, T. R.; Lee, M. S.; Lee, A. M.; Gwaltney, S. R.; Adams, T. R.; Ochsenfeld, C.; Gilbert, A. T. B.; Kedziora, G. S.; Rassolov, V. A.; Maurice, D. R.; Nair, N.; Shao, Y.; Besley, N. A.; Maslen, P. E. Dombroski, J. P.; Daschel, H.; Zhang, W.; Korambath, P. P.; Baker, J.; Byrd, E. F. C.; Van Voorhis, T.; Oumi, M.; Hirata, S.; Hsu, C.-P.; Ishikawa,
N.; Florian, J.; Warshel, A.; Johnson, B. G.; Gill, P. M. W.; Head-Gordon, M.; Pople, J. A. J. Comput. Chem. 2000, 21, 1532-1548.

(44) Runge, E.; Gross, E. Phys. Rev. Lett. 1999, 96, 1533.

(45) Casida, M. E. Recent Advances in Density Functional Methods, Part I; Chong, D. P., Ed.; World Scientific: Singapore, 1995.

(46) van Gisbergen, S. J. A.; Groeneveld, J. A.; Rosa, A.; Snijders, J. G.; Baerends, E. J. J. Phys. Chem. A 1999, 103, 6835-6844.

(47) Rosa, A.; Baerends, E. J.; van Gisbergen, S. J. A.; van Lenthe, E.; Groeneveld, J. A.; Snijders, J. G. J. Am. Chem. Soc. 1999, 121, 1035610365.

(48) Serra, L.; Rubio, A. Phys. Rev. Lett. 1997, 78, 1428. 